\title{
El análisis multimodal del anuncio publicitario audiovisual para el aula de Lengua Castellana y Literatura en Educación Secundaria y Bachillerato
}

\author{
Zósimo López Penai(i) \\ Universidad Internacional de La Rioja, Logroño, La Rioja, España
}

\begin{abstract}
Resumen
El objetivo de este artículo consiste en proponer una metodología para el análisis y la comprensión de mensajes publicitarios en el aula de Lengua Castellana y Literatura durante la Educación Secundaria y el Bachillerato en España. Dada la complejidad semiótica de este tipo de mensajes, en el presente trabajo se plantea la posibilidad de llevar a cabo un análisis de tipo multimodal que permita desentrañar los diferentes modos que integran el mensaje y llegar a alcanzar así su significado de conjunto. Para lograr este objetivo, se expondrá, en primer lugar, un apartado en el que se apuntará la necesidad de encontrar nuevas formas de análisis para las distintas formas de publicidad. Posteriormente, se propondrá un marco teórico en torno al concepto de multimodalidad y el tipo de análisis que deriva de esta disciplina. Finalmente, se plantearán las bases teóricas sobre las que hacer un análisis multimodal de un anuncio audiovisual.
\end{abstract}

Palabras clave

Publicidad. Análisis de contenido. Educación Secundaria. Lengua.

\section{A análise multimodal da publicidade audiovisual para a aula}

de Língua Espanhola e Literatura no Ensino Secundário e no Ensino Médio

\begin{abstract}
Resumo
O objetivo deste artigo é propor uma metodologia para a análise e compreensão das mensagens publicitárias na aula de Língua Espanhola e Literatura durante o Ensino Secundário e o Ensino Médio na Espanha. Dada a complexidade semiótica desse tipo de mensagem, este trabalho propõe a possibilidade de realizar uma análise multimodal que permita desvendar os diferentes modos que compõem a mensagem e, assim, alcançar o seu significado global. Para alcançar este objetivo, será apresentada primeiro uma seção na qual será salientada a necessidade de encontrar novas formas de análise para as diferentes formas de publicidade. Em seguida, será proposto um quadro teórico em torno do conceito de multimodalidade e do tipo de análise que deriva dessa disciplina. Finalmente serão propostas as bases teóricas sobre as quais fazer uma análise multimodal de um anúncio audiovisual.
\end{abstract}

\section{Palavras-chave}

Publicidade. Análise de conteúdo. Ensino Secundário. Língua. 
The multimodal analysis of the audiovisual advertisement for the Spanish

Language and Literature classroom in Secondary and Baccalaureate

Education

\begin{abstract}
The aim of this article is to propose a methodology for the analysis and understanding of advertising messages in the Spanish Language and Literature classroom during Secondary Education and High School in Spain. Given the semiotic complexity of this type of message, this paper proposes the possibility of carrying out a multimodal analysis that will allow us to unravel the different modes that make up the message and thus reach its overall meaning. In order to achieve this objective, we will first present a section in which we will point out the need to find new forms of analysis for the different forms of advertising. Then, we will propose a theoretical framework around the concept of multimodality and the type of analysis that derives from this discipline. Finally, we will propose the theoretical bases on which to make a multimodal analysis of an audiovisual advertisement.
\end{abstract}

\title{
Keywords
}

Advertising. Content analysis. Secondary Education. Language.

\section{Introducción}

La publicidad es un fenómeno comunicativo en el que estamos continuamente inmersos como consecuencia de nuestra incesante exposición a los diversos medios de comunicación. Esta circunstancia se ha acentuado en una era en la que cada ciudadano tiene acceso a Internet y, por tanto, a alguna forma de comunicación mediante distintos medios. A este respecto, podemos traer a colación los datos que ofrece el Instituto Nacional de Estadística (INE) en su Encuesta sobre equipamiento y uso de tecnologías de la información y la comunicación en los hogares del año 2019. Con respecto a la franja de edad que nos interesa en el presente artículo, esto es, aquella que comprende entre los 12 y los 18 años, se señala que "[...] el uso de Internet es una práctica mayoritaria en los jóvenes de 16 a 24 años, con un 99,2\% en los hombres y un 99,0\% en las mujeres" (INE, 2019 , s.p.). En el mismo informe podemos leer que un 92,6\% del total de personas entre 16 y 24 años de edad encuestadas afirman utilizar Internet varias veces al día. En cuanto al análisis del tipo de actividad realizada en Internet, este informe señala que participar en redes sociales, usar mensajería instantánea y buscar información son las acciones más frecuentes. Importa indicar, asimismo, que un 92,9\% del total de niños entre los 10 y los 15 
años encuestados afirman ser usuarios de Internet en los últimos tres meses. Todas las señaladas pueden suponer acciones en las que se podría acceder a distintas formas de publicidad. Estos datos nos ofrecen un primer argumento para apoyar la necesidad de explicar y enseñar a analizar el mensaje publicitario desde edades tempranas.

Asimismo, resulta necesario considerar que la publicidad puede afectar al desarrollo del alumnado en tanto que ciudadano, ya que este tipo de comunicación comercial puede imponer criterios morales, éticos, culturales y/o incluso estéticos (MARTíNEZ, 1994). Tenemos que tener en cuenta que la publicidad no solo está relacionada con la venta de productos o servicios, sino que también aporta conocimientos para la construcción de la identidad sociocultural de los ciudadanos; así pues, si bien estamos delante de un discurso de venta, nuestros alumnos están también expuestos ante una muy eficaz herramienta ideológica de alienación social (LOMAS, 2001). Otras cuestiones que debemos considerar a la hora de valorar la importancia de que nuestros estudiantes conozcan el lenguaje publicitario son las actitudes y valores que desprenden sus mensajes. Según Santacruz y Camacho (2003, p. 144), podrían sintetizarse en las siguientes:

[...] exaltación del individualismo, conformismo social, obsesión por el éxito, primacía de la cultura de la apariencia, ética de corte hedonista que busca la satisfacción en el placer y en el presente, concepción ahistórica de la realidad, mitificación de la juventud y culto al cuerpo, el sexo, la difusión de estereotipos y la violencia.

Si tenemos en cuenta las razones expuestas, parece evidente la importancia que adquiere la didáctica de la publicidad en el aula de Educación Secundaria Obligatoria y Bachillerato en España (AMBRÓS I PALLARÉS, 2006; ARCONADA, 2006; MARTÍNEZ, 1994; MORO, 2011). De hecho, la disciplina aparece contemplada de distintas formas en diversas materias del currículo de este nivel educativo (ESPAÑA, 2015; MORO, 2011). Así, si bien es cierto que el discurso publicitario puede ser descrito, interpretado, analizado y trabajado desde diferentes áreas del saber, el profesorado de Lengua Castellana y Literatura en Educación Secundaria tiene una responsabilidad particular en la enseñanza de este tipo de mensaje persuasivo por el peso que la publicidad adquiere en los diferentes bloques de contenidos de la materia Lengua Castellana y Literatura incluidos en el currículo oficial de Enseñanza Secundaria y Bachillerato en España (LÓPEZ PENA, 2017). 
El objetivo de este artículo consiste en proponer una serie de guías teóricas que sirvan de ayuda al profesorado de Enseñanza Secundaria para comprender el mensaje publicitario y así poder explicar este tipo de comunicación comercial al alumnado. Esta tarea se vertebra sobre dos ideas principales:

- La primera tiene que ver con que la publicidad consiste en una forma de comunicación compleja compuesta por varios y diferentes tipos de mensajes emitidos en distintos medios. Por este motivo, necesitamos de una sistematización que favorezca su decodificación para lograr comprenderla y analizarla.

- La segunda se relaciona con la manera de desgranar la información por medio de un análisis basado en la semiótica multimodal, que podría representar un nuevo enfoque desde el cual enfrentarse a los análisis de mensajes semióticamente complejos, esto es, aquellos que adquieren significado solo como un conjunto organizado de distintos modos tal y como se explicará más adelante.

Para alcanzar el objetivo propuesto, aportaremos:

- En primer lugar, la metodología sobre la cual asentamos nuestro trabajo, que parte de la necesidad de encontrar nuevas formas de análisis para las distintas formas de Publicidad en el aula de Secundaria y se basa en la multimodalidad y el tipo de análisis que emana de esta disciplina.

- En segundo lugar, se expondrán los principales resultados, que consisten en una propuesta de análisis multimodal para un producto publicitario; en este caso, de un anuncio audiovisual. Cerraremos el trabajo con algunas consideraciones finales en torno a las diferentes cuestiones tratadas a lo largo del texto.

\section{Metodología}

\subsection{Nuevas formas para el análisis de la comunicación publicitaria audiovisual: la alfabetización multimodal}

Los mensajes publicitarios pueden adquirir distintas formas en función del público objetivo al que vaya dirigida la campaña. Las modalidades son heterogéneas y 
están, además, en constante evolución. Estos tipos de mensajes están compuestos por una densidad de signos que quizás otros mensajes, como los estrictamente periodísticos, no presentan. Así pues, constituyen mensajes semióticamente complejos, tal y como antes hemos señalado.

Dado que disponemos solo del espacio de un artículo, para el presente trabajo hemos optado por centrarnos en una forma concreta de publicidad - la de tipo audiovisual - y sobre ella propondremos un modelo de análisis. Este está basado en la teoría semiótica multimodal (JEWITT; KRESS, 2003; KRESS, 2000, 2003, 2010; KRESS; VAN LEEUWEN, 1996, 2001, 2002; VAN LEEUWEN, 2005) y adaptado a lo que estimamos pertinente para un nivel de alumnado que cursa la materia de Lengua Castellana y Literatura en Educación Secundaria y Bachillerato. Creemos que este modelo puede ayudar al personal docente a favorecer y facilitar el análisis y la comprensión de la complejidad del mensaje publicitario a su alumnado. Este tipo de análisis puede resultar interesante en muchos sentidos, pero principalmente lo es porque tiene una gran capacidad a la hora de desgranar unidades mínimas de significado de diferente naturaleza (audio, visual, audiovisual, multimedia, etc.) que interactúan a la vez dentro de una entidad comunicativa de intencionalidad publicitaria, como, por ejemplo, puede ser un advergame o un spot televisivo.

Actualmente, en un mundo digital nos enfrentamos a diferentes entornos multimodales: pensemos, por ejemplo, en las distintas pantallas de los dispositivos desde los cuales accedemos a Internet. De acuerdo con esta circunstancia, tenemos que tener en cuenta las maneras de representación de algo lo suficientemente moldeado como para ser aprendido y cómo esto va a ser enseñado. Por este motivo, una mejor comprensión sobre lo que se va a enseñar y lo que se va a aprender en un entorno multimodal de un aula actual de Enseñanza Secundaria y Bachillerato es crucial para explorar las maneras de representación por medio de todos los modos que pueden ser tratados en un mensaje. En este sentido, es posible que el punto principal de la multimodalidad, para nuestros intereses como docentes, sea la representación y sistematización de los significados de un mensaje. Obviamente esta manera de descripción y análisis de un mensaje y, por tanto, este proceso de alfabetización multimodal tienen que ser enseñados y aprendidos en la clase por medio de la figura del docente. 


\subsection{La multimodalidad y su aplicación}

La multimodalidad es una disciplina que se preocupa del significado que se crea a través de las diferentes configuraciones y combinaciones que pueden tener en un mismo mensaje la imagen, el gesto, la mirada, la postura corporal, el sonido, la escritura, la tipografía, la música o el discurso, entre otros elementos. Aunque el nacimiento de la multimodalidad encuentra su génesis en autores como Kress y van Leeuwen (1996), estos investigadores beben de las fuentes del lingüista de origen británico Michael Alexander Kirkwood Halliday, quien desarrolla la llamada Lingüística Sistémico-Funcional (HALLIDAY, 1978; HALLIDAY; MATTHIESSEN, 2004) y se basa en un enfoque en el que la lengua es una fuente semiótica la cual es modelada por sus usuarios en los contextos de la cultura y de la situación.

El término "multimodalidad" tiene que ver con la interrelación de los denominados "modos" - la imagen, la acción, los gestos, la ropa o la música, por mencionar solo algunos - como un conjunto organizado de fuentes semióticas para la creación de significado. Un "modo" es una fuente socialmente modelada y culturalmente dada para crear un significado (KRESS, 2009). Una banda sonora, un gesto, una portada de un periódico, un personaje en una obra teatral, un color determinado pueden ser ejemplos de modos utilizados para la representación y comunicación de una serie de significados y valores dentro de un contexto cultural determinado. Precisamente, el objetivo de un análisis de semiótica social es la identificación y descripción de los modos y fuentes semióticas que están disponibles en una situación dada. De esta manera, es posible ver cómo la gente utiliza esas fuentes, qué elecciones hace de ellas y cuáles son sus motivaciones para escoger unas fuentes en lugar de otras (JEWITT; BEZEMER; O'HALLORAN, 2016).

Debemos tener en cuenta que todos los modos - incluyendo los modos lingüísticos de escritura, habla, gestos, mirada, tiempo o distancia personal, entre otros posibles - contribuyen a la construcción de significado de diferentes maneras y, por esta razón, ningún modo puede quedar aislado en un análisis multimodal, ya que todos y cada uno de ellos - además de su contexto - actúan en interdependencia entre sí dentro de un sistema diseñado para decodificar un significado de una entidad comunicativa. La comprensión de la alfabetización multimodal exige la investigación de todos los modos 
como un conjunto que interviene en un evento comunicativo $\mathrm{y}$, de este modo, debe enfocarse en el aula (JEWITT, 2008).

\subsubsection{El análisis multimodal}

El propósito de los análisis e investigaciones multimodales es la comprensión de los principios de los usos y de las fuentes modales disponibles en una representación multimodal, como puede ser, por ejemplo, un anuncio publicitario audiovisual. Este método de investigación examina cómo la gente crea significados a través de la selección de los recursos semióticos más adecuados de un conjunto de modos disponible para el emisor del mensaje en un emplazamiento geográfico y temporal determinado (JEWITT, 2009; MACHIN, 2007). Para alcanzar esa comprensión de significado, el análisis multimodal persigue el significado de un momento comunicativo en vez de buscar fijar un inventario universal para un modo, ya que elementos como el contexto situacional o el contexto cultural compartido determinan la óptima codificación de un mensaje.

Un punto relevante en el que se centra el análisis multimodal es la sistematización de la manera en la que los recursos comunicativos son utilizados por la gente en un contexto social dado o, remitiéndonos a las teorías de Kress y van Leeuwen (1996, 2001), el significado como un constructo social. Esto implica descubrir una serie de datos para conocer las decisiones que toma un hablante sobre los diferentes usos que puede tener un modo para así marcar las relaciones entre el lenguaje y el contexto social. El contexto social es el concepto de "marco" (BATEMAN; SCHMIDT, 2012; GOFFMAN, 2012) como el espacio social donde se construyen los significados y los valores. Este cobra una importancia capital a la hora de diseñar un análisis multimodal, sobre todo si pensamos en un análisis multimodal de un producto cultural como puede ser un spot publicitario. Si lo aplicamos a la disciplina semiótica, el concepto "marco" alude al fragmento de nuestra realidad que discriminamos.

Hasta el momento, hemos revisado los conceptos de modo y fuente semiótica; a ellos deben sumarse las llamadas posibilidades modales (JEWITT, 2009) y, por tanto, conviene detenerse y reflexionar sobre la importancia que pueden tener los elementos diegéticos a la hora de seleccionar los modos. Estas posibilidades modales podemos definirlas como lo que es posible expresar y representar sencillamente; esto es, cómo un 
modo lleva siendo utilizado repetidamente para representar algo por medio de convenciones sociales en contextos determinados y que, de manera inequívoca, remite a los mismos significados. Tal y como antes hemos señalado, tanto un film como un anuncio publicitario audiovisual $u$ otros muchos elementos comunicativos pueden tomarse como una unidad susceptible de ser analizada. Estas unidades, compuestas por numerosos modos y fuentes semióticas, podemos denominarlos genéricamente artefactos multimodales (HIIPPALA, 2013) y, según esta explicación, podemos afirmar que un anuncio publicitario (sea este audible, visual, audiovisual, háptico, olfativo, etc.) es un artefacto multimodal. En esta ocasión concreta nos interesa ahondar, como antes hemos indicado, en la decodificación semiótica de uno de tipo producto audiovisual.

2.2.2 ¿Cómo hacer un análisis multimodal de un producto audiovisual?: el caso de la publicidad audiovisual

Pese a que varios autores habían hecho ya una aproximación sistemática al análisis multimodal de fragmentos de productos audiovisuales (O'HALLORAN, 2004), en el año 2012 Bateman y Schmidt publicaron el manual Multimodal film analysis: how films mean para el análisis multimodal de filmes. Estos autores hacen un extenso estado de la cuestión sobre los diferentes análisis audiovisuales aplicados al lenguaje cinematográfico tomando como punto de partida a Bordwell (1982, 1985, 1989, 2005, 2007) o Metz (1966, 1974). La tradición del análisis fílmico está marcada profundamente por las escuelas e ideologías sobre todo desde una perspectiva marxista y/o una influencia psicoanalítica.

Otra tradición importante para el análisis fílmico es la que proviene de la narratología. Por su parte, Bateman y Schmidt (2012) proponen una segmentación para el análisis multimodal de productos fílmicos basada en preceptos estructuralistas de corte lingüístico aludiendo a los ejes paradigmático y sintagmático. Estos autores establecen como la unidad mínima de significado de un producto audiovisual el fotograma, que funcionaría como unidad sintáctica dentro de un eje sintagmático que avanza en el tiempo. Esta forma de trabajo va a ayudarnos a la hora de establecer elementos concretos para realizar un análisis multimodal de las unidades que componen un relato audiovisual.

En lo que se refiere al análisis de publicidad audiovisual, se pueden encontrar diversos métodos de análisis, entre los que se encuentran los comentarios de textos 
fílmicos y las descripciones, en mayor o menor medida, con una carga de subjetividad. Muchos de los análisis, sobre todo iconográficos o semióticos, parten de las teorías de Panofsky, quien, en 1939, editaba su trabajo titulado Studies in iconology, una extensa labor de iconología que continuaba el trabajo que Cesare Ripa había comenzado en el siglo XVI. La propuesta de Panofsky (1939) se fundamentaba en una estratificación de capas de significado que, aun hoy, se sigue utilizando en diferentes análisis de productos audiovisuales publicitarios. Son también frecuentes los análisis de contenido basados en la tradición narratológica, como se aludió anteriormente en lo referido a los análisis fílmicos, en combinación con disciplinas como la semiótica, la iconología, el análisis del discurso y la retórica. Esta última se emplea de manera desvirtualizada y utilizando simplemente una batería de figuras retóricas.

De este modo, en muchos casos, se olvidan los modos de organización del discurso (dispositio) o la ejecución técnica del mismo (actio). Por su parte, en el campo de los estudios de corte filológico sobre la publicidad, es habitual encontrar trabajos que se centran solamente en la parte literaria, lingüística y textual, olvidando el contexto, el medio, la imagen fija, la imagen en movimiento, el sonido o la música. En este sentido, podemos considerar que, si bien no existe una metodología perfecta para llevar a cabo el análisis de un anuncio publicitario, pensamos que el multimodal puede ayudar a sistematizar la información contenida en este tipo de artefactos multimodales y, así, facilitar la completa comprensión de estos tipos de mensajes semióticamente complejos (O'HALLORAN; TAN; KWAN LIN, 2013).

Sentadas estas bases, pasamos a proponer una serie de fases de cara al diseño de un análisis multimodal de un anuncio publicitario audiovisual con la intención de facilitar el trabajo al personal docente de Lengua Castellana y Literatura de Enseñanza Secundaria y Bachillerato.

\section{Resultados: fases propuestas para realizar un análisis multimodal de un anuncio publicitario en el aula de Educación Secundaria}

Cuando llega el momento de explicar el discurso y el lenguaje publicitario en el aula, es necesario alcanzar un panorama global y completo sobre esta manera de comunicación (LÓPEZ PENA, 2017). Para lograr este objetivo, se debe proceder a la 
configuración del diseño del análisis del anuncio publicitario en formato audiovisual. Esta tarea puede llevarse a cabo a través de las distintas fases que a continuación exponemos en la tabla siguiente.

Tabla 1 - Fases para la realización de un análisis multimodal de un spot audiovisual (continúa)

\begin{tabular}{|c|c|c|}
\hline $1^{\text {a Fase }}$ & $\begin{array}{c}\text { Marcar el contexto } \\
\text { del spot }\end{array}$ & $\begin{array}{l}\text { A este propósito se realizaría un acopio de documentación sobre } \\
\text { todo los elementos relativos al artefacto multimodal que queremos } \\
\text { analizar y su contexto. Esta labor ayudaría a conocer factores como: } \\
\text { - La intencionalidad. } \\
\text { - La situación. } \\
\text { - El registro lingüístico y comunicativo. } \\
\text { - Los participantes en el evento comunicativo. }\end{array}$ \\
\hline $2^{\mathrm{a}}$ Fase & $\begin{array}{l}\text { Definir los modos y } \\
\text { los descriptores }\end{array}$ & $\begin{array}{l}\text { En este punto se trata de reflexionar sobre qué es lo que realmente } \\
\text { queremos analizar de este artefacto multimodal. } \\
\text { 1. La primera cuestión que tenemos que tener en cuenta es si el } \\
\text { artefacto multimodal es estático o dinámico. A este respecto, } \\
\text { Bateman (2013) introduce un término apropiado para esta } \\
\text { cuestión, que es la diferenciación o establecimiento de rasgos } \\
\text { específicos que definen a un artefacto multimodal o a otro. Para } \\
\text { dicha caracterización, este autor aporta el concepto de género y } \\
\text { multimodalidad (BATEMAN, 2013). La primera observación que } \\
\text { hace, en este sentido, es que no es lo mismo el análisis de un } \\
\text { artefacto multimodal estático que la de un artefacto multimodal } \\
\text { no dinámico, sino que, según la naturaleza de estos artefactos, } \\
\text { la metodología de análisis puede variar sensiblemente. } \\
\text { 2. En algunos casos quizás tengamos que analizar un contexto } \\
\text { social de emplazamiento: pensemos, por ejemplo, en el análisis } \\
\text { de una pintada de cuartos de baño o de un cartel que anuncia } \\
\text { un festival de música popular; pero en otros quizás debamos } \\
\text { analizar los medios de comunicación donde se visiona dicho } \\
\text { artefacto: pensemos en un film, en un videoclip o en un } \\
\text { anuncio, el tipo de artefacto que más nos interesa aquí. } \\
\text { 3. Algo semejante podemos indicar con respecto a la selección de } \\
\text { los modos que pueden ser casi infinitos, porque infinita puede } \\
\text { llegar a ser nuestra curiosidad; así pues, en esta fase debemos } \\
\text { acotar dentro de lo posible los diferentes modos a tratar. } \\
\text { 4. Cuando escogemos los modos, acto seguido debemos } \\
\text { diseccionar los diferentes descriptores de los que se va a } \\
\text { componer este modo. De esta manera, si definimos un modo en } \\
\text { un análisis de un artefacto multimodal audiovisual como } \\
\text { "Personaje 1", entonces debemos establecer los descriptores } \\
\text { que nos van a interesar de cada uno de los personajes: estos } \\
\text { podrían ser, entre otros, vestuario, diálogo, lenguaje corporal, } \\
\text { cronémica o proxémica. }\end{array}$ \\
\hline $3^{a}$ Fase & $\begin{array}{l}\text { Preparar una } \\
\text { plantilla para hacer } \\
\text { un inventario de } \\
\text { los elementos } \\
\text { escogidos que } \\
\text { aparecen en los } \\
\text { modos }\end{array}$ & $\begin{array}{l}\text { - Llegados a este punto cabe mencionar que existe un software } \\
\text { específico para realizar un análisis multimodal de imágenes, } \\
\text { sitios web y productos audiovisuales (JEWITT, 2006; LIM FEI et } \\
\text { al., 2015; O'HALLORAN et al., 2008, 2014a, 2014b; ROHLFING } \\
\text { et al., 2006), aunque implica cierto coste económico y un proceso } \\
\text { de aprendizaje para utilizarlo. }\end{array}$ \\
\hline
\end{tabular}

Educação \& Formação, Fortaleza, v. 5, n. 3, e2839, set./dez. 2020

DOI: https://doi.org/10.25053/redufor.v5i15set/dez.2839

https://revistas.uece.br/index.php/redufor/index 
Tabla 1 - Fases para la realización de un análisis multimodal de un spot audiovisual

(conclusión)

\begin{tabular}{|c|c|c|}
\hline $3^{a}$ Fase & $\begin{array}{c}\text { Preparar una } \\
\text { plantilla para hacer } \\
\text { un inventario de } \\
\text { los elementos } \\
\text { escogidos que } \\
\text { aparecen en los } \\
\text { modos }\end{array}$ & $\begin{array}{l}\text { - Para poder acercar esta metodología a todo tipo de aulas, } \\
\text { proponemos la utilización de cualquier programa informático } \\
\text { que deje trabajar en celdas. Más tarde, con ayuda de este } \\
\text { software, configuraremos una parrilla en la que estableceremos } \\
\text { dos grandes macromodos: uno en el que situaremos los modos } \\
\text { estáticos -modos que permanecen inalterables durante una } \\
\text { escena - y otro en el que situaremos los modos dinámicos - } \\
\text { modos que cambian durante la escena. Una vez determinados } \\
\text { los macromodos, procedemos a disgregar los modos y estos en } \\
\text { descriptores. }\end{array}$ \\
\hline $4^{\mathrm{a}}$ Fase & $\begin{array}{l}\text { Segmentar el spot } \\
\text { en unidades } \\
\text { mínimas, esto es, } \\
\text { en los planos de } \\
\text { las secuencias }\end{array}$ & $\begin{array}{l}\text { Para esto, extraemos un fotograma de un plano para emplazarlo en } \\
\text { nuestra parrilla y así dispondremos de una referencia gráfica para el } \\
\text { análisis de los diferentes modos. } \\
\text { Cuando se trata de planos secuencia donde los cuadros varían, } \\
\text { proponemos extraer un fotograma por cada cambio de cuadro. }\end{array}$ \\
\hline $5^{\mathrm{a}}$ Fase & $\begin{array}{l}\text { Completar las } \\
\text { celdas } \\
\text { correspondientes a } \\
\text { cada descriptor }\end{array}$ & $\begin{array}{l}\text { Una vez que hemos determinado el número de modos, el número de } \\
\text { descriptores, la duración de las secuencias y que, asimismo, } \\
\text { podemos extraer los planos y plasmarlos en un fotograma que } \\
\text { podemos insertar en el espacio de nuestra parrilla destinada para tal } \\
\text { fin, completamos las celdas correspondientes a cada descriptor. }\end{array}$ \\
\hline $6^{a}$ Fase & $\begin{array}{l}\text { Completar los } \\
\text { descriptores }\end{array}$ & $\begin{array}{l}\text { A la hora de completar los descriptores sucede que siempre vamos } \\
\text { aportar una visión parcial y subjetiva sobre los mismos. Una manera } \\
\text { de mitigar esta subjetividad sería tener la posibilidad de obtener un } \\
\text { grupo de control heterogéneo que hubiera podido aportar diferentes } \\
\text { visiones sobre un mismo objeto. Dada la dificultad de llevar a cabo } \\
\text { este propósito en un aula de Secundaria, puede resultar más } \\
\text { funcional proponer el uso de un software gratuito de reconocimiento } \\
\text { visual tal como Google API Vision o Microsoft Azure. El apoyo de los } \\
\text { datos obtenidos mediante esta aplicación nos ayuda a complementar } \\
\text { los datos obtenidos en los descriptores previamente establecidos. En } \\
\text { el caso de Google API Vision, esta nos proporciona datos como el } \\
\text { estado de ánimo denotado en la expresión facial de personajes } \\
\text { humanos o humanizados. Debe de considerarse, en todo caso, que } \\
\text { en el caso de personajes de animación personificados no reconoce a } \\
\text { día de hoy las expresiones faciales y sus sentimientos } \\
\text { correspondientes. Google asigna a los elementos que se encuentran } \\
\text { en la imagen (figuras, lugares, animales, especies, etc.) una serie de } \\
\text { sellos o atributos, aporta también correspondencias con otros sites } \\
\text { en Internet y que están indexados por Google, indica se existe texto } \\
\text { en la imagen, cuál es la paleta de colores que aparece en la imagen } \\
\text { y los colores dominantes o mismo si es una imagen posiblemente } \\
\text { acomodada a un público infantil-juvenil o no. Estos tipos de datos } \\
\text { complementan de una manera importante el diseño de investigación } \\
\text { sobre un artefacto multimodal y, por esta razón, pensamos que son } \\
\text { una herramienta considerablemente útil para el fin que perseguimos. }\end{array}$ \\
\hline $7^{a}$ Fase & $\begin{array}{l}\text { Sistematizar y } \\
\text { analizar los datos } \\
\text { obtenidos }\end{array}$ & $\begin{array}{l}\text { Una vez completados todos los espacios destinados a los } \\
\text { descriptores correspondientes en cada celda, obtendremos un } \\
\text { corpus estandarizado de términos y datos tanto en cantidad como en } \\
\text { calidad para poder comenzar el análisis del artefacto multimodal } \\
\text { audiovisual que nos permita acceder a su significado global. }\end{array}$ \\
\hline
\end{tabular}

Fuente: Elaboración propia (2020).

Educação \& Formação, Fortaleza, v. 5, n. 3, e2839, set./dez. 2020

DOI: https://doi.org/10.25053/redufor.v5i15set/dez.2839

https://revistas.uece.br/index.php/redufor/index 
Estas fases se han probado en otras experiencias para el análisis de productos audiovisuales, como en largometrajes, con un resultado satisfactorio (LÓPEZ PENA, 2018). Asimismo, las distintas fases indicadas ayudan a la sistematización y posterior análisis de contenidos y significado que un producto audiovisual puede representar, facilitando así la labor del docente de Lengua Castellana y Literatura en Educación Secundaria y Bachillerato para el análisis de spots publicitarios.

\section{Conclusiones}

En el presente artículo, hemos apuntado la constante exposición a distintas formas de publicidad del alumnado de Secundaria y Bachillerato. Además de esta situación, hay que considerar que la comprensión y valoración crítica del mensaje publicitario, su intención comunicativa, así como la comprensión de los elementos verbales y no verbales son mencionados en diversos lugares del currículum de Lengua Castellana y Literatura en sus distintas etapas (ESPAÑA, 2015). Esas circunstancias derivan en la necesidad de que este aprenda a desentrañar correctamente el significado de los mensajes de esta naturaleza.

Importa, considerar, no obstante, la complejidad semiótica de los mensajes publicitarios, puesto que estos son unidades integradas por diferentes signos. Su análisis debe abordarse, pues, desde una metodología de conjunto que logre alcanzar el significado de cada uno de esos elementos así como su significado de conjunto o global. Solo así el docente podrá comprender y enseñar a analizar en el aula este tipo de mensaje publicitario. En este sentido, en el presente trabajo se ha propuesto, desde un punto de vista teórico, un método de análisis multimodal así como sus distintas fases. A través de este sería posible analizar los principios de los usos de las fuentes modales disponibles en una representación multimodal, del que puede ser ejemplo un anuncio publicitario audiovisual, el caso escogido para este artículo.

De este modo, tanto el docente como el alumnado podrán llegar a comprender el significado que se crea a través de la combinación de la imagen, el gesto, las animaciones, los colores, el discurso o la música, entre otros, en un mismo mensaje que pertenece a un contexto situacional, social y cultural. Pensamos que este método favorecerá el mejoramiento de la competencia mediática de los estudiantes en una era 
en la cual los contenidos digitales por dispositivos móviles son los imperantes y que, bajo nuestro punto de vista, sería necesario llevar ejemplos de esta naturaleza al aula de lengua para analizarlos y decodificarlos.

\section{Referencias}

AMBRÓS I PALLARÉS, A. La educación en comunicación en el área de lengua de la Educación Secundaria Obligatoria del MEC y Cataluña. ¿Un malentendido?. Lenguaje y Textos, Valencia, v. 23-24, p. 149-168, 2006.

ARCONADA, M. Á. Cómo trabajar con la publicidad en el aula: competencia comunicativa y textos publicitarios. Barcelona: Graó, 2006.

BATEMAN, J. Multimodal analysis of film within the gem framework. Ilha do Desterro, Florianópolis, v. 64, p. 49-84, 2013.

BATEMAN, J.; SCHMIDT, K.-H. Multimodal film analysis: how films mean. New York/London: Routledge, 2012.

BORDWELL, D. Figures traced in light: on cinematic staging. Los Angeles/London: University of California, 2005.

BORDWELL, D. Making meaning: inference and rhetoric in the interpretation of Cinema. Cambridge: Harvard University, 1989.

BORDWELL, D. Narration in the fiction film. Madison: University of Wisconsin, 1985.

BORDWELL, D. Poetics of cinema. London/New York: Routledge, 2007.

BORDWELL, D. Textual analysis. Enclitic, New York, v. 6, n. 1, p. 125-136, 1982.

ESPAÑA. Real Decreto 1105/2014, de 26 de diciembre, por el que se establece el currículo básico de la Educación Secundaria Obligatoria y del Bachillerato. Boletín Oficial del Estado, Madrid, n. 3, 3 ene. 2015.

GOFFMAN, E. La presentación de la persona en la vida cotidiana. Buenos Aires: Amorrortu, 2012.

HALLIDAY, M. A. K. Language as social semiotic. London: Edward Arnold, 1978.

HALLIDAY, M. A. K.; MATTHIESSEN, C. M. I. M. An introduction to functional grammar. London: Edward Arnold, 2004.

HIIPPALA, T. Modelling the structure of a multimodal artefact. Helsinki: Universidad de Helsinki, 2013. Disponible en: https://helda.helsinki.fi/handle/10138/41736. Acceso el: 20 abr. 2020. 
INE - Instituto Nacional de Estadística. Encuesta sobre equipamiento y uso de tecnologías de información y comunicación en los hogares. 2019. Disponible en: https://www.ine.es/ss/satellite?l=es_es\&amp;c=ineseccion_c\&amp;cid=1259925528782\&

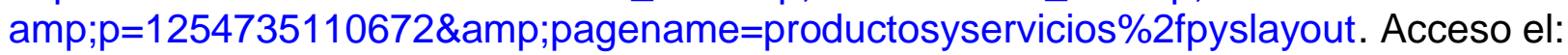
21 feb. 2020.

INE - Instituto Nacional de Estadística. Equipamiento y uso de TIC en los hogares. 2019. Disponible en:

https://www.ine.es/dyngs/inebase/es/operacion.htm?c=estadistica_c\&cid=125473617674 1\&menu=ultidatos\&idp=1254735976608. Acceso el: 20 abr. 2020.

JEWITT, C. Multimodality and literacy in school classrooms. Review of Research in Education, New York, v. 32, n. 1, p. 241-267, 2008.

JEWITT, C. Technology, literacy and learning: a multimodal approach. London: Routledge, 2006.

JEWITT, C. (Ed.). The Routledge handbook of multimodal analysis. London: Routledge, 2009.

JEWITT, C.; BEZEMER, J.; O'HALLORAN, K. Introducing multimodality. London/New York: Routledge, 2016.

JEWITT, C.; KRESS, G. (Ed.). Multimodal literacy. New York: Peter Lang, 2003.

KRESS, G. Literacy in the new media age. London: Routledge, 2003.

KRESS, G. Multimodality. In: KALANTZIS, M.; COPE, B. (Ed.). Multiliteracies: literacy learning and the design of social futures. Routledge: London, 2000. p. 182-202.

KRESS, G. Multimodality: a social semiotic approach to contemporary communication. London: Routledge, 2010.

KRESS, G. What is mode? In: CAREY, J. (Ed.). The Routledge handbook of multimodal analysis. London: Routledge, 2009. p. 54-67.

KRESS, G.; VAN LEEUWEN, T. Colour as a semiotic mode: notes for a grammar of colour. Visual Communication, New York, v. 1, n. 3, s.p., 2002.

KRESS, G.; VAN LEEUWEN, T. Multimodal discourse: the modes and media of contemporary communication. London: Edward Arnold, 2001.

KRESS, G.; VAN LEEUWEN, T. Reading images: the grammar of visual design. London: New York: Routledge, 1996.

LIM FEI, V. et al. Teaching visual texts with the multimodal analysis software. Educational Technology Research and Development, v. 63, p. 915-935, 2015. 
LOMAS, C. La estética de los objetos y la ética de los sujetos. Comunicar, Huelva, v. 17, p. 31-39, 2001.

LÓPEZ PENA, Z. Aproximación à análise multimodal de produtos audiovisuais: o filme The Way. In: SEMINARIO-ASAMBLEA INTERNACIONAL ASOCIACIÓN DE ESTUDIOS SOBRE DISCURSO Y SOCIEDAD. 2., 2018, Vigo. Conferencia... Vigo: Ediso, 2018. DOI: 10.13140/RG.2.2.21710.36169.

LÓPEZ PENA, Z. La publicidad en el aula de Lengua Castellana y Literatura en Enseñanza Secundaria en España: retos y desafíos. Lenguaje y Textos, Valencia, v. 45, p. 89-99, 2017.

MACHIN, D. Introduction to multimodal analysis. London: Bloomsbury, 2007.

MARTÍNEZ, E. La publicidad y su integración en las aulas. Comunicar, Huelva, v. 3. p. 6573, 1994.

METZ, C. Film language: a semiotics of the cinema. Oxford/Chicago: Chicago University, 1974.

METZ, C. La grande syntagmatique du film narratif. Communications, Paris, v. 8, p. 120124, 1966.

MORO, M. M. La publicidad en los curricula de las aulas escolares. 2011. Disponible en: http://www.vamosapublicidad.com/wp-content/uploads/2011/10/publi-en-curricula.pdf. Acceso el: 3 feb. 2020.

O'HALLORAN, K. Visual semiosis in film. In: O'HALLORAN, K. L. (Ed.). Multimodal discourse analysis: systemic-functional perspectives. London: England; New York: Continuum, 2004. p. 109-131.

O'HALLORAN, K. et al. Challenges and solutions to multimodal analysis: technology, theory and practice. In: FANG, Y.; WEBSTER, J. (Ed.). Developing systemic functional linguistics: theory and application. London: Equinox, 2014a. p. 271-297.

O'HALLORAN, K. et al. Challenges in designing digital interfaces for the study of multimodal phenomena. Information Design Journal, v. 18, n. 1, p. 2-12, 2008.

O'HALLORAN, K. et al. Multimodal analytics: software and visualization techniques for analyzing and interpreting multimodal data. In: JEWITT, C. (Ed). The Routledge handbook of multimodal analysis. London: Routledge, 2014b. p. 386-396.

O'HALLORAN, K.; TAN, S.; KWAN LIN, M. E. 'Above All': the myth of 'dreams' as advertising tool. In: PENNOCK-SPECK, B.; SAZ RUBIO, M. M. (Ed.). The multimodal analysis of television commercials. Valencia: Universitat de València, 2013. p. 113-135.

PANOFSKY, E. Studies in iconology. New York: Oxford University, 1939. 
ROHLFING, K. et al. Comparison of multimodal annotation tools: workshop report. Gesprächsforschung, v. 7, n. 7, p. 99-123, 2006.

SANTACRUZ, F. J.; CAMACHO, A. La publicidad: una experiencia en el aula. Comunicar, Huelva, v. 20, p. 142-146, 2003.

VAN LEEUWEN, T. Introducing social semiotics. London: Routledge, 2005.

Zósimo López Pena, Universidad Internacional de La Rioja (UNIR), Departamento de Didáctica de Lengua y Literatura, Facultad de Educación

iDhttps://orcid.org/0000-0001-6058-9003

Profesor de Didáctica de la Lengua en la UNIR, licenciado en Filología Hispánica por la Universidad de Santiago de Compostela (USC). Diploma de Estudios Avanzados en Lingüística y doctor en Comunicación e Industrias Creativas por la USC. Desde 2004 ha desarrollado actividades laborales vinculadas a la docencia, a la investigación y a la publicidad. Ha sido profesor visitante en la Universidad de Verona y en la Universidade do Minho. Colabora desde el año 2016 con el grupo de investigación Galabra (Estudios de los Sistemas Culturales Gallego, Luso, Brasileño y Africanos de Lengua Portuguesa) de la Universidad de Santiago de Compostela. Sus líneas de investigación se centran en la comunicación en lengua española, la didáctica de la comunicación publicitaria en lengua española, análisis del discurso y multimodalidad.

Contribución de autoría: Desarrolló toda la investigación y escritura.

E-mail: zosimo.lopez@unir.net

Editora responsable: Lia Machado Fiuza Fialho Pareceristas ad hoc: Marta Camarero-Figuerola y Gustavo Flórez-Mojica

\section{Cómo citar este artículo (ABNT):}

LÓPEZ PENA, Zósimo. El análisis multimodal del anuncio publicitario audiovisual para el aula de Lengua Castellana y Literatura en Educación Secundaria y Bachillerato. Educ.

Form., Fortaleza, v. 5, n. 3, p. 1-16, 2020. Disponible en:

https://revistas.uece.br/index.php/redufor/article/view/2839.

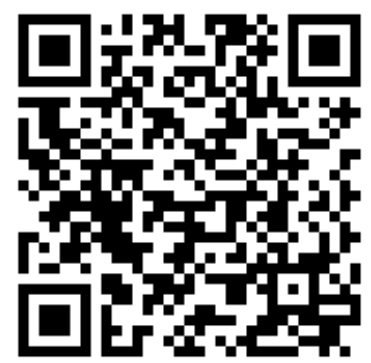

Recibido el 27 de febrero de 2020.

Aceptado el 29 de marzo de 2020.

Publicado el 27 de mayo de 2020.

Educação \& Formação, Fortaleza, v. 5, n. 3, e2839, set./dez. 2020

DOI: https://doi.org/10.25053/redufor.v5i15set/dez.2839

https://revistas.uece.br/index.php/redufor/index 